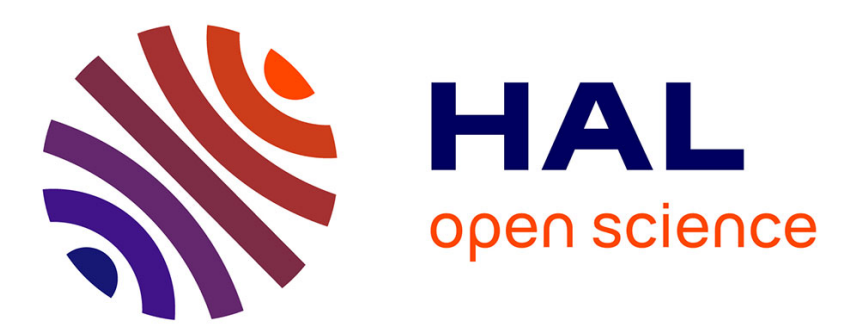

\title{
A practical methodology to screen oil recovery processes involving spontaneous imbibition
}

T. Chevalier, J. Labaume, A. Delbos, T. Clemens, V. M Waeger, B. Bourbiaux, M. Fleury

\section{- To cite this version:}

T. Chevalier, J. Labaume, A. Delbos, T. Clemens, V. M Waeger, et al.. A practical methodology to screen oil recovery processes involving spontaneous imbibition. Transport in Porous Media, 2019, 127 (3), pp.729-743. 10.1007/s11242-018-01229-z . hal-02117744

\section{HAL Id: hal-02117744 \\ https://hal-ifp.archives-ouvertes.fr/hal-02117744}

Submitted on 2 May 2019

HAL is a multi-disciplinary open access archive for the deposit and dissemination of scientific research documents, whether they are published or not. The documents may come from teaching and research institutions in France or abroad, or from public or private research centers.
L'archive ouverte pluridisciplinaire HAL, est destinée au dépôt et à la diffusion de documents scientifiques de niveau recherche, publiés ou non, émanant des établissements d'enseignement et de recherche français ou étrangers, des laboratoires publics ou privés. 


\title{
A practical methodology to screen oil recovery processes involving spontaneous imbibition
}

\author{
T. Chevalier ${ }^{1 *}$, J. Labaume $^{1}$, A. Delbos ${ }^{1}$, T. Clemens ${ }^{2}$, V. M. Waeger ${ }^{2}$, B. Bourbiaux ${ }^{1}$, M. Fleury ${ }^{1}$ \\ ${ }^{1}$ IFP Energies nouvelles, 1 - 4 avenue de Bois-Préau 92852 Rueil-Malmaison, France \\ ${ }^{2}$ OMV, Aktiengesellschaft Trabrennstraße 6-8 1020 Vienna, Austria \\ *thibaud.chevalier@ifp.fr
}

\begin{abstract}
We present a general novel technique to monitor saturation changes on small rock samples of only $15 \mathrm{~mm}$ in diameter and $20 \mathrm{~mm}$ in length for the purpose of assessing the kinetics of spontaneous imbibition processes. With a fully-3D imbibition configuration involving countercurrent flows through all faces of the sample, the method is based on a NMR technique in which the sole oil phase present within the sample is monitored. The experimental method is fast for two reasons that are i) the possibility to perform accurate measurements on tiny samples, ii) the adoption of a 3D flow geometry. The kinetics of oil desaturation during spontaneous imbibition is analyzed with the help of an analytical 3D diffusion model, according to which the kinetics is proportional to the value of a "capillary" diffusion coefficient. For the purpose of demonstrating our methodology, we used this technique to compare the spontaneous imbibition of restored sandstone miniplugs from a sandstone reservoir, with and without alkali in the imbibing brine. The imbibition kinetics was quantified as capillary diffusion coefficient values. The studied case results revealed mixed impacts of alkali on the spontaneous imbibition kinetics, involving both a brine-oil interfacial tension change and a wettability alteration of the rock, the latter requiring further investigation beyond the scope of this article.
\end{abstract}

Keywords: imbibition, NMR, diffusion, chemical recovery, wettability

\section{List of symbols}

\begin{tabular}{|c|c|}
\hline EOR & Enhanced oil recovery \\
\hline IFT & Interfacial tension $[\mathrm{N} / \mathrm{m}]$ \\
\hline NMR & Nuclear magnetic resonance \\
\hline $\mathrm{D}, 2 \mathrm{r}$ & Diameter of the core (r: radius) [m] \\
\hline $\mathrm{D}_{\mathrm{c}}$ & Capillary diffusion coefficient $\left[\mathrm{m}^{2} / \mathrm{s}\right]$ \\
\hline $\mathrm{D}_{\mathrm{e}}$ & Effective molecular diffusion coefficient $\left[\mathrm{m}^{2} / \mathrm{s}\right]$ \\
\hline $\mathrm{D}_{\mathrm{m}}$ & Molecular diffusion coefficient $\left[\mathrm{m}^{2} / \mathrm{s}\right]$ \\
\hline $\mathrm{K}$ & Single-phase permeability $\left[\mathrm{m}^{2}\right]$ \\
\hline$k_{r w}, k_{r o}$ & Relative permeabilities to water (w) and oil (o) \\
\hline $\mathrm{L}, 21$ & Length of the core (l: half length) [m] \\
\hline$\lambda_{\mathrm{w},} \lambda_{\mathrm{o}}$ & Relative mobility for water (w) and oil (o) $\left[\mathrm{Pa}^{-1} \cdot \mathrm{s}^{-1}\right]$ \\
\hline$\mu_{w}, \mu_{o}$ & Viscosities of water (w) and oil (o) [Pa.s] \\
\hline $\mathrm{Pc}$ & Capillary pressure $[\mathrm{Pa}]$ \\
\hline$\Phi$ & Porosity of the core \\
\hline $\mathrm{S}_{\mathrm{w}}, \mathrm{S}_{\mathrm{o}}$ & Water (w) and oil (o) saturation \\
\hline $\mathrm{S}_{\mathrm{w}} *, \mathrm{~S}_{\mathrm{o}} *$ & Scaled water (w) and oil (o) saturation (cf eq (3)) \\
\hline
\end{tabular}




$\begin{array}{ll}\mathrm{S}_{\mathrm{wi}}, \mathrm{S}_{\mathrm{oi}} & \text { Initial water (w) and oil (o) saturation } \\ \mathrm{S}_{\mathrm{of}} & \text { Final oil saturation } \\ \mathrm{T}_{2} & \text { Transverse relaxation time }[\mathrm{s}] \\ \mathrm{V} & \text { Bulk volume of the sample }\left[\mathrm{m}^{3}\right] \\ \mathrm{V}_{\mathrm{p}} & \text { Porous volume }\left[\mathrm{m}^{3}\right] \\ \mathrm{V}_{\mathrm{o}} & \text { Oil volume in the core }\left[\mathrm{m}^{3}\right] \\ \mathrm{V}_{\mathrm{oi}} & \text { Initial oil volume in the core }\left[\mathrm{m}^{3}\right]\end{array}$

\section{ACKNOWLEDGEMENTS}

We thank OMV for allowing us to publish the results. Wolfgang Hujer is also acknowledged for providing laboratory data and rock description.

\section{INTRODUCTION}

The measurement and analysis of spontaneous displacements of oil by water has been the subject of numerous research studies since the sixties (Mattax and Kyte 1962, Hamon and Vidal 1986, 1986, Bourbiaux and Kalaydjian 1990). Although the scaling laws are well known (Shouxiang et al. 1997, Schmid and Geiger 2012), it is no surprise to find recent work on the subject because it is a non-linear complex problem. Essentially, there is an attempt to derive flow parameters (capillary pressure, relative permeability) from saturation vs. time curve (Haugen et al. 2014, Alyafei and Blunt 2018) with either local or average saturation data. For this purpose, analytical or numerical analysis is necessary and approximate analytical solutions have been found in one dimension and before the invading phase reaches the end of the core (Ruth et al. 2007).

Herein, our objective is to describe a methodology to evaluate the EOR efficiency of chemicals from NMR measurements of spontaneous imbibition of chemical solutions on small cores. As imbibition processes are well known to be time consuming, the experiments are considerably shortened when using small samples, as will be detailed later. However, with standard methods, saturation data cannot be collected with enough accuracy; hence, we propose a low field NMR technique to monitor very small porous volumes. Performing 3D instead of 1D experiments also contributes to shortening experiments duration.

Our methodology also includes a fast analytical screening of experimental imbibition kinetics results based on the saturation vs. time curves, without having to resort to advanced numerical simulations at that preliminary stage of EOR process selection. Actually, EOR process modeling is complicated by the occurrence of multiple physical mechanisms that may enhance imbibition. Spontaneous imbibition can be enhanced or influenced by wettability changes generated by properly designed chemicals (Hirasaki and Zhang 2004, Chabert et al. 2010, Bourbiaux et al. 2016), by low salinity brines (Morrow and Buckley 2013, Sheng 2014), or by the natural surfactants resulting from reactive crude oils in the presence of alkaline solutions (Cooke et al. 1974). The reaction of alkali with some components of oil can, indeed, lead to the formation of natural soaps that reduce the interfacial tension between oil and brine.

For demonstration purpose, the proposed methodology is applied to an alkaline imbibition process envisaged in actual field context. Small restored plugs from the St Ulrich field in the 
Vienna Basin were subjected to spontaneous imbibition experiments with two brines differing by the absence or presence of alkali. For reservoir rock-fluids systems, the possible benefit of an alkaline injection is expected to result from two phenomena (Ehrlich et al. 1974, Campbell and Krumrine 1979). First, the brine composition changes (including $\mathrm{pH}$ ) may result in rock wettability alteration mechanisms, similar to the ones invoked for low-salinity flooding effects. The second mechanism stems from the reaction of acid and/or basic oil components with alkali leading to the formation of in-situ surfactants (soaps) that can alter the water-oil interfacial tension, and again also the wettability of the rock. Spontaneous imbibition, that is driven by the capillary pressure curve, is a sensitive indicator of both effects of alkali, that are the decrease of brine-oil interfacial tension and rock surface property changes.

In this study, reservoir samples were first aged with reservoir oil at irreducible saturation, then they were subjected to the spontaneous imbibition either of a reference injection brine, or of an alkaline brine. The observed difference between respective imbibition kinetics was used as a criterion to assess the impact of alkaline additive on the oil recovery. Thanks to our low-field NMR saturation monitoring method, small rock samples (15 $\mathrm{mm}$ in diameter) could be used instead of plugs of standard diameter $(40 \mathrm{~mm})$, which was helpful as the low permeability of the studied rock let us expect a slow imbibition kinetics. Saturation data were recorded at time intervals varying from 1 hour up to 1 day. Then, the recovery kinetics was analyzed with the analytical diffusion model evoked before, and capillary diffusion coefficients were determined as a characteristic of the imbibition kinetics in the absence and in the presence of alkaline additive. Specific implementation of the NMR technique for accurate saturation measurement, the capillary diffusion approach of spontaneous imbibition, and the scaling of imbibition experiments are discussed in the course of the paper as they constitute the major contributions of our experimental EOR screening methodology.

\section{MATERIAL and METHODS}

\subsection{Samples and fluids}

\subsubsection{Brine recombination}

Two brine compositions were considered in relation with the field operating conditions: the reservoir connate brine composition and the composition of the brine used for field waterflooding (Table 1). The latter brine is denoted as the injection brine in the following.

Three synthetic brines were reconstituted on the basis of these two compositions as explained hereafter. The reservoir connate brine composition was only used to restore the initial saturation of rock samples, that is oil in the presence of irreducible brine. The injection brine composition was used to perform the reference imbibition experiment. Its ionic composition is similar to that of the connate brine but less concentrated. Alkaline imbibition experiments were performed with the injection brine composition in which we removed the initial divalent ions and added sodium carbonate at a concentration of $15 \mathrm{~g} / \mathrm{l}$ (divalent ions were removed to avoid precipitation). Note that to remove the signal of the water from the NMR spectrum, $\mathrm{D}_{2} \mathrm{O}$ is used for the brine preparation instead of $\mathrm{H}_{2} \mathrm{O}$. The brines compositions are summarized in Table 1. 
Table 1: Composition of the different brines used in the experiments ; connate brine at Swi, injection brine for imbibition experiments on samples 1a and 2a, and alkaline brine for experiments on samples $1 \mathrm{~b}$ and $2 \mathrm{~b}$.

\begin{tabular}{|l|c|c|c|}
\hline \multirow{2}{*}{$\begin{array}{c}\text { Synthetic brine } \\
\text { composition }\end{array}$} & \multicolumn{3}{|c|}{ Concentration (g/L) } \\
\cline { 2 - 4 } $\mathrm{NaCl}$ & Connate brine & Injection brine & Alkaline brine \\
\hline $\mathrm{KCl}$ & 15 & 10.5 & 10.5 \\
\hline $\mathrm{MgCl} 2$ & 0.145 & 0.09 & 0.09 \\
\hline $\mathrm{CaCl} 2$ & 0.52 & 0.28 & \\
\hline $\mathrm{Na} 2 \mathrm{CO} 3$ & 0.5 & 0.34 & 15 \\
\hline
\end{tabular}

\subsubsection{Oil and interfacial tensions}

Crude oil samples were collected from the producing well Saint Ulrich 65. The samples were analysed in terms of oil-water interfacial tension (IFT) to verify that no additive was present (Hirasaki and Zhang 2004) in the crude oil. Its water content was also measured using the Karl Fischer method, and checked to be low. API gravity, viscosity and density of that crude oil were determined to be respectively $31^{\circ}$ API, $8.2 \pm 0.1 \mathrm{cP}$ (at $100 \mathrm{~s}^{-1}$ and $40^{\circ} \mathrm{C}$ ) and $0.854 \pm 0,001$ $\mathrm{g} / \mathrm{cm}^{3}$ (at $40^{\circ} \mathrm{C}$ ). The oil was then filtered at the reservoir temperature to avoid any plugging during the injection in porous media. The interfacial tension (IFT) was measured using the Wilhelmy plate method at $40^{\circ} \mathrm{C}$ (Figure 1). For the oil-alkaline brine system, the IFT is much lower $(0.8 \mathrm{mN} / \mathrm{m})$ than the one of the oil-injection brine system $(8 \mathrm{mN} / \mathrm{m})$. This is an expected effect since the hydroxyl anions present in the alkaline brine may react with some polar components of the oil to form soaps that may be considered as natural surfactants.

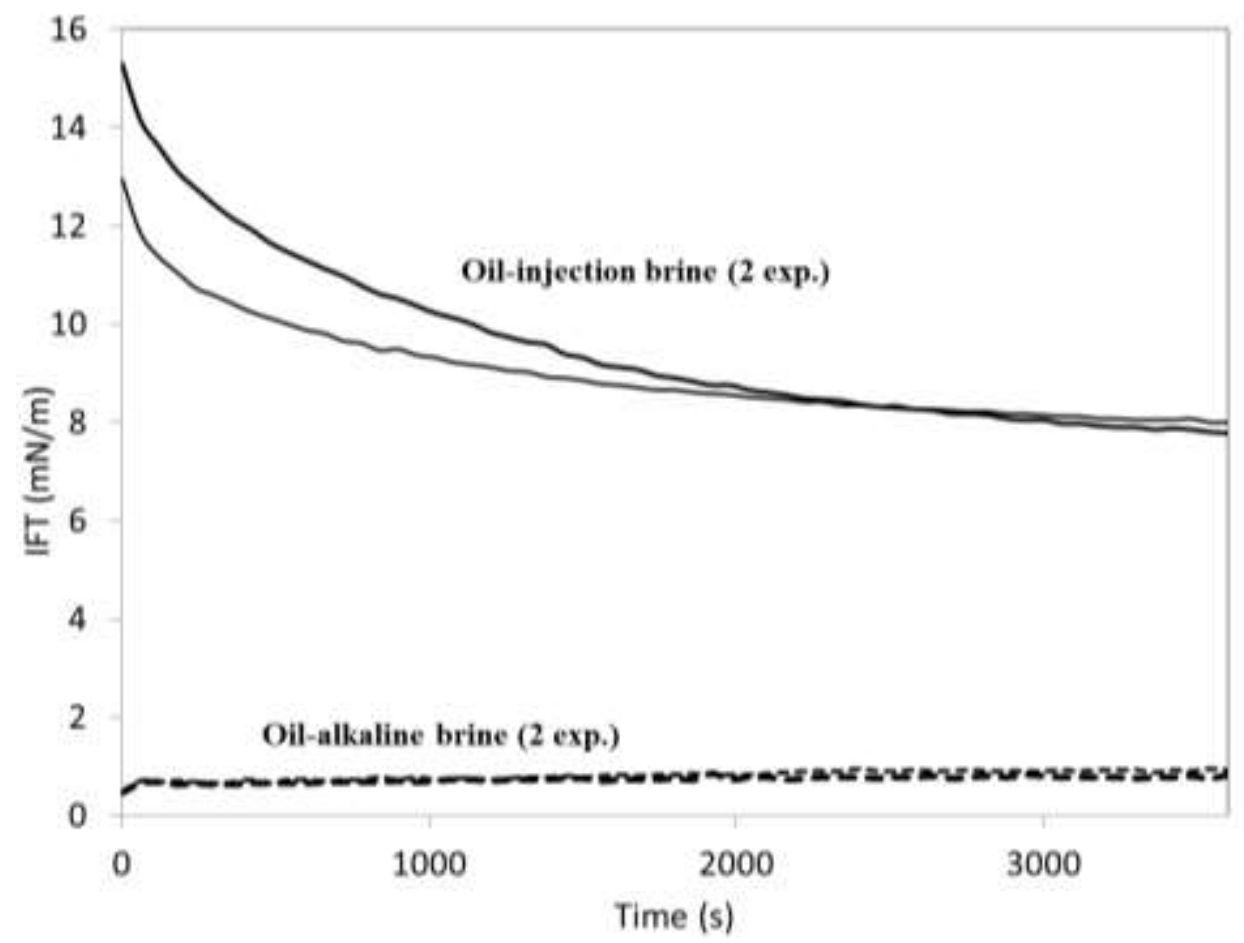

Figure 1 : Interfacial tension, determined by Wilhelmy plate method at $40^{\circ} \mathrm{C}$, between oil and the injection brine (full lines) and alkaline brine (dashed lines). Experiments were repeated twice. 


\subsubsection{Plug selection and restoration}

Small cylindrical samples ("plugs") have been drilled from turbiditic Flysch sandstones of Eocene age. The Flysch in Austria constitutes a fractured siliciclastic basement reservoir. Flow paths are mainly provided by fractures whereas oil storage capacity is present in the matrix. The matrix rock is a fine-to-medium-grained, moderately well-sorted sandstones. Mineralogical composition is dominated by quartz, with minor feldspar and low volumes of glauconite. The samples chosen for imbibition have not been used for porosity-permeability measurements. Matrix porosity and permeability from a nearby drilled plug are respectively $8.3 \%$ and $0.3 \mathrm{mD}$. Reservoir properties of the matrix are mainly deteriorated by compaction and quartz cementation. To select samples as homogeneous as possible, plugs were scanned as received. Four sister plugs among the most homogeneous ones were selected for the study.

Small cylindrical samples were cored through these plugs, dried and saturated with the connate brine. They were then set at Swi using centrifuge. Once at Swi, they were aged for 3 weeks at reservoir temperature. During that aging period, cores were flooded every week with one pore volume of dead oil. This aging time is considered adequate to restore the in-situ wettability.

\subsection{NMR experimental method}

The experimental set-up described in detail elsewhere (Chevalier et al. 2018) is briefly summarized here. The spontaneous imbibition is done in a glass tube of outer diameter $18 \mathrm{~mm}$ closed with a plastic cap to prevent evaporation of the brine during the process as shown in Figure 2. Imbibition cells remain in an oven at $40^{\circ} \mathrm{C}$ except during the measurements performed in the NMR spectrometer at $30^{\circ} \mathrm{C}$ (a MARAN $23.7 \mathrm{MHz}$ from Oxford Instruments equipped with a $18 \mathrm{~mm}$ diameter probe). The experiments lasted two weeks and the interval between two measurements varied between $30 \mathrm{~min}$ at the beginning of the process and several hours at the end. This frequency of measurement is designed to capture the rapid evolution of the saturation during the first day of imbibition, followed by a much slower kinetics afterwards.

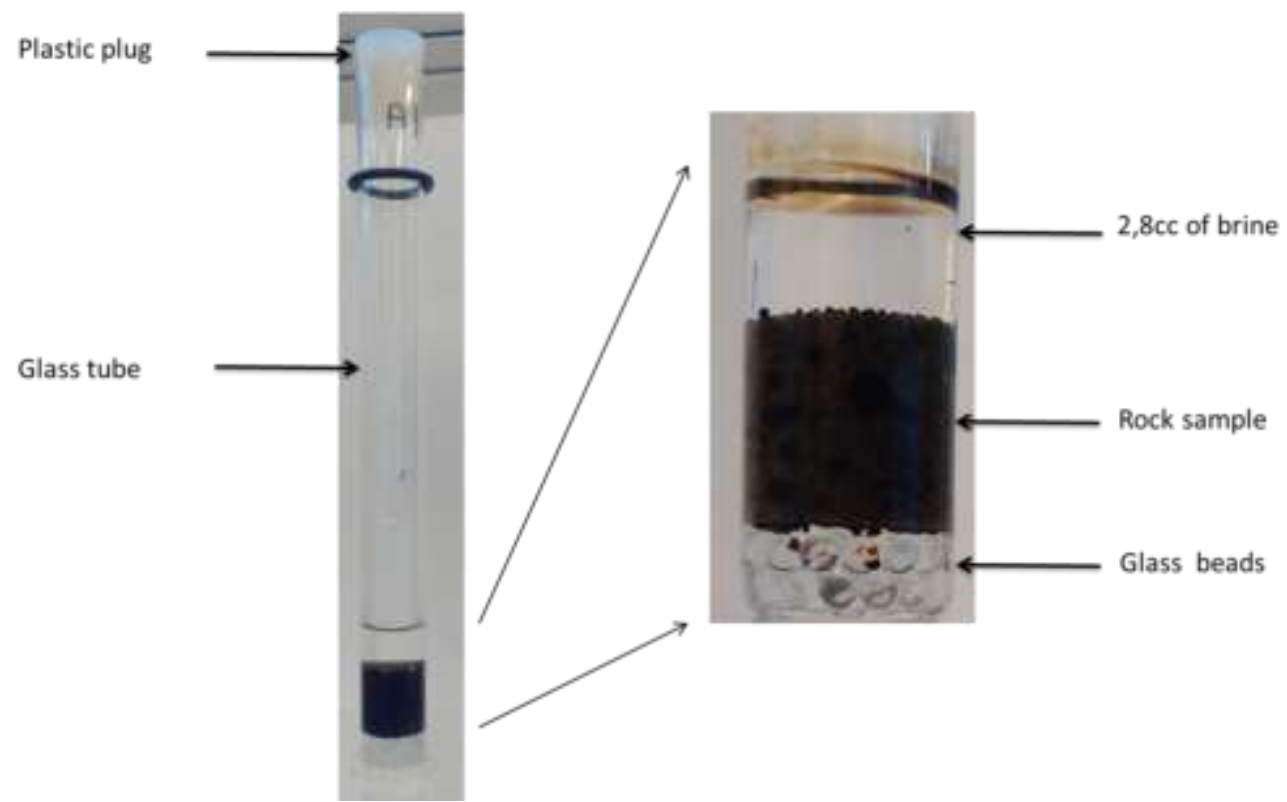

Figure 2 : Imbibition tube placed in the NMR spectrometer (outer diameter $18 \mathrm{~mm}$ ). 
In these experiments, the aqueous solution imbibes through all the faces of the sample. As spontaneous imbibition process is known to have a slow kinetics, it is advantageous to work with small samples: the NMR technique is crucial, making possible the detection of some hundreds of micro-liter in the sample with reasonable accuracy around $\pm 10 \mu \mathrm{L}$ (Table 2). During the spontaneous imbibition, oil is expelled out of the sample and segregates at the top of the surrounding $\mathrm{D}_{2} \mathrm{O}$ brine above the volume investigated by the NMR probe. By using deuterium instead of hydrogen in the brine, all the measured NMR signal emanates from the oil held in the sample and we can directly infer the oil saturation, without needing to separate oil and water in $\mathrm{T}_{2}$ distributions. However, $\mathrm{T}_{2}$ distributions were needed to eliminate the contribution of oil droplets sticking to the surface of the sample (Figure 2) and to determine the exact oil saturation of the miniplug. We explain below how we corrected the data by applying a cutoff at $100 \mathrm{~ms}$ on the $\mathrm{T}_{2}$ distribution to remove the droplet contribution.

Table 2: Samples characteristics (dimensions, porosity and porous volume), brine type, initial and final saturation. Capillary diffusion coefficient $\left(D_{c}\right)$ from diffusion analysis (see text and Figure 6).

\begin{tabular}{|l|l|l|l|l|l|l|l|l|l|l|l|}
\hline \multicolumn{3}{|l|}{ Sample } & \multicolumn{3}{l|}{ Initial } & \multicolumn{2}{l|}{ Recovery } & \\
\hline $\mathrm{n}^{\circ}$ & $\mathrm{L}$ & $\mathrm{D}$ & $\Phi$ & $\mathrm{V}_{\mathrm{p}}$ & $\begin{array}{l}\text { Brine } \\
\text { type }\end{array}$ & $\mathrm{V}_{\mathrm{oi}}$ & $\mathrm{S}_{\mathrm{wi}}$ & $\Delta \mathrm{V}_{\mathrm{o}}$ & $\begin{array}{l}\Delta \mathrm{V}_{\mathrm{o}} / \\
\mathrm{V}_{\mathrm{oi}}\end{array}$ & $\mathrm{S}_{\mathrm{of}}$ & $\mathrm{D}_{\mathrm{c}}$ \\
\hline & {$[\mathrm{mm}]$} & {$[\mathrm{mm}]$} & {$[\%]$} & {$[\mu \mathrm{L}]$} & & {$[\mu \mathrm{L}]$} & & {$[\mu \mathrm{L}]$} & & & {$\left[\mathrm{cm}^{2} / \mathrm{s}\right]$} \\
\hline $1 \mathrm{a}$ & 18.3 & 14.7 & 10.0 & 310 & Injection & 277 & $11 \%$ & 123 & $44 \%$ & $50 \%$ & $1.210^{-6}$ \\
\hline $1 \mathrm{~b}$ & 18.1 & 14.7 & 10.0 & 307 & Alkaline & 265 & $14 \%$ & 117 & $44 \%$ & $48 \%$ & $6.010^{-7}$ \\
\hline $2 \mathrm{a}$ & 17.1 & 14.7 & 10.2 & 295 & Injection & 246 & $17 \%$ & 109 & $44 \%$ & $46 \%$ & $9.810^{-7}$ \\
\hline $2 \mathrm{~b}$ & 18.2 & 14.7 & 9.1 & 282 & Alkaline & 261 & $8 \%$ & 114 & $44 \%$ & $52 \%$ & $6.610^{-7}$ \\
\hline
\end{tabular}

To determine the cut-off of $100 \mathrm{~ms}$, we compared the oil volumes obtained at the end of the imbibition experiment and after wiping off the droplets sticking on the surface, more precisely, the corresponding $\mathrm{T}_{2}$ distribution are different (Figure 3 ) with the distribution mode shifted to a larger value (around $200 \mathrm{~ms}$ ) for the last point compared to the end point (around $100 \mathrm{~ms}$ ). This shift is significant and in agreement with the distribution obtained on bulk oil (Figure 3) for which the mode is also around $200 \mathrm{~ms}$; however, the difference between bulk oil (oil droplets) and oil inside the porous media is not large enough to be resolved on the distribution and hence, a cut-off approach was adopted and applied on the entire time series. Based on these observations, a cutoff at $100 \mathrm{~ms}$ could be applied to the $\mathrm{T}_{2}$ distributions in order to quantify the quantity of oil solely saturating the pores of the miniplug. In the following, the imbibition kinetic data drawn from NMR measurements are analyzed on the basis of the flow equations that underlie spontaneous imbibition and whose theoretical basis is recalled hereafter. 

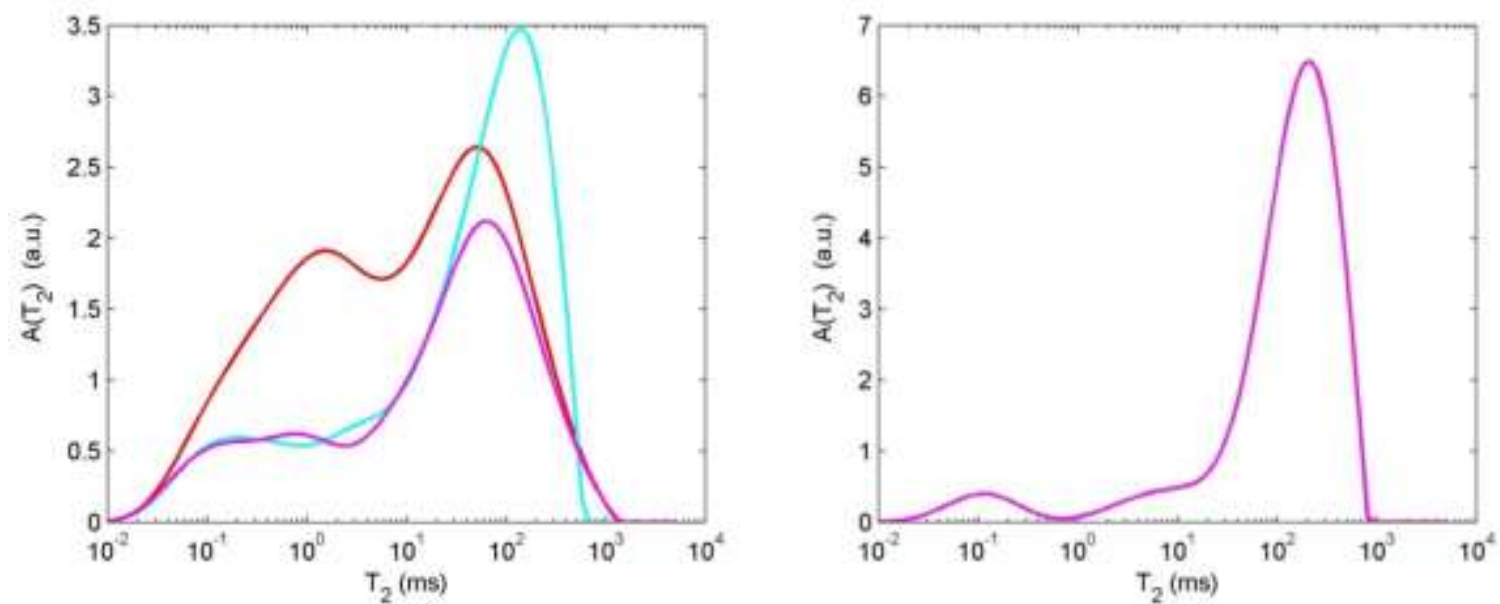

Figure 3: Left: the three $T_{2}$ distributions for samples $1 b$ at initial time of imbibition (red), at the end of imbibition before wiping off droplets (light blue) and at the end of imbibition after wiping off droplets(pink). Right: $T_{2}$ distribution for bulk oil.

\section{THEORETICAL BASIS OF RESULTS ANALYSIS}

Darcy's law governing two phase flow in porous media can be written in terms of a unique convection-diffusion equation (Marle 1981, Chavent and Jaffre 1986). The spontaneous imbibition of very small samples as the ones used in this study is mainly driven by capillary forces with negligible buoyancy effects. That working assumption can be verified by calculating the ratio between the initial (maximum) gravity head applied on the miniplug and the capillary pressure within the miniplug. A characteristic value of that capillary pressure was read from the Pc curve shown in Figure 4, for the water saturation interval covered during imbibition, i.e. $\left[S_{w i}\right.$, $\left.1-S_{\text {of }}\right]$. Miniplug height and water-oil density contrast were considered to evaluate the maximum initial gravity head. The so-calculated buoyancy-to-capillarity ratio is respectively equal to 0.025 and 0.25 for the tests without and with alkali. That is, buoyancy did not much influence the spontaneous process which can then be considered a capillary(-driven) invasion process.

From a mathematical view point, generalized Darcy law and mass balance enable one to formulate the countercurrent flow of two immiscible fluids, water and oil, as the following equation (Chavent and Jaffre 1986, Stoll et al. 2008, Schmid and Geiger 2012, Foley et al. 2017) :

$$
\Phi \frac{\partial S_{w}}{\partial t}=-\nabla\left[K \frac{\lambda_{w} \lambda_{o}}{\lambda_{t}} \nabla P_{c}\right] \text { with } \lambda_{o}=\frac{k_{r o}}{\mu_{o}}, \lambda_{w}=\frac{k_{r w}}{\mu_{w}}, \lambda_{t}=\lambda_{o}+\lambda_{w}
$$

in which $\mathrm{K}$ is the single-phase permeability, $S_{\mathrm{w}}$ is the water saturation, $\lambda_{\mathrm{w}}, \lambda_{\mathrm{o}}$ are the relative mobility for oil (o) and water (w), $k_{r w}, k_{r o}$ the relative permeabilities to water and oil and $\mu_{w}, \mu_{o}$ the viscosities of water and oil respectively. Porosity is assumed constant. $\mathrm{k}_{\mathrm{rw}}, \mathrm{k}_{\mathrm{ro}}$ and Pc are functions of the water saturation. By differentiating Pc with respect to saturation, one can rewrite above equation as a non-linear diffusion equation:

$$
\frac{\partial S_{w}}{\partial t}=D_{c} \Delta S_{w} \quad \text { with } D_{C}=\frac{1}{\phi} K \frac{\lambda_{w} \lambda_{o}}{\lambda_{t}} \frac{\partial P_{c}}{\partial S_{w}}
$$


with $D_{c}$ the variable (saturation-dependent) diffusion coefficient. $D_{c}$ is called "capillary diffusion" coefficient to emphasize that capillary forces are presumably the driving forces in the present study. The term "diffusion" refers to the mathematical form of Eq. 2, although "capillary dispersion" is also used by some authors (Alyafei et al. 2016, Schmid et al. 2016). Many developments of that mathematical form of diffusion equation are used in the literature to study countercurrent transfers (Tecklenburg et al. 2013, March et al. 2016, Zhou et al. 2017).

If $\mathrm{D}_{\mathrm{c}}$ is assumed constant, then analytical solutions of diffusion equation 2 are available (Crank 1976), in particular for the 3D cylindrical geometry corresponding to the experimental situation. The evolution with time of the scaled oil saturation $\mathrm{S}_{\mathrm{o}} *$ is the product of two solutions $\left(\mathrm{C}_{\mathrm{ps}}\right.$ for the plane sheet and $\mathrm{C}_{\mathrm{cyl}}$ for the infinite cylinder) and is given by:

$$
\begin{gathered}
S_{o}^{*}=C_{p s} C_{c y l} \quad \text { with } \quad S_{o}^{*}=\frac{S_{o}-S_{o f}}{S_{o i}-S_{o f}} \\
C_{p s}=\sum_{n=0}^{\infty} \frac{8}{(2 n+1)^{2} \pi^{2}} \exp \left(-D_{c}(2 n+1)^{2} \pi^{2} \frac{t}{4 l^{2}}\right) \\
C_{c y l}=\sum_{n=1}^{\infty} \frac{4}{r^{2} q_{n}^{2}} \exp \left(-D_{c} q_{n}^{2} t\right)
\end{gathered}
$$

where $\mathrm{q}_{\mathrm{n}}$ are the positive roots of the equation $J_{0}\left(r q_{n}\right)=0, J_{0}$ being the first type Bessel function of order zero, $r$ is the radius of the cylinder and $2 l$ its length. $S_{o i}$ and $S_{\text {of }}$ are respectively the initial and final oil saturation at the beginning and the end of spontaneous imbibition. The boundary condition associated with the above solution is a constant "saturation" outside the sample $\left(S_{\mathrm{w}}\right.$ =1). This model has been used successfully in the past (Berne et al. 2010) to analyze deuterium exchange experiments solely governed by molecular diffusion of water within a cylindrical porous medium in the context of very low permeability porous media.

For the case of spontaneous experiments, the assumption of constant diffusion coefficient $\mathrm{D}_{\mathrm{c}}$ for solving equation 2 is a significant simplification and is worth examining further. This coefficient depends obviously on saturation and is therefore not spatially constant within the sample. For this purpose and as in a previous work (Stoll et al. 2008), we calculated $D_{c}$ with standard $k_{r}$ functions of the saturation and an empirical imbibition Pc function. The latter took into account the actual pore size distribution of the porous medium under consideration, as inferred from a mercury injection curve. The coefficient $\mathrm{D}_{\mathrm{c}}$ was found to be nearly constant over a large saturation range (Figure $4, \mathrm{Sw}^{*} \in[0.2-0.8]$ ) and to decrease sharply outside this range (the $\mathrm{D}_{\mathrm{c}}$ value close to experimental ones is coincidental). Hence, the theoretical solution given above, that involves a constant $D_{c}$, is expected to fit approximately the experimental data, independently of experimental inaccuracies. The approximate fit of a single $D_{c}$ value is nevertheless sufficient to characterize the overall kinetics recorded during a given imbibition experiment, as will be shown next from the results of application case. 


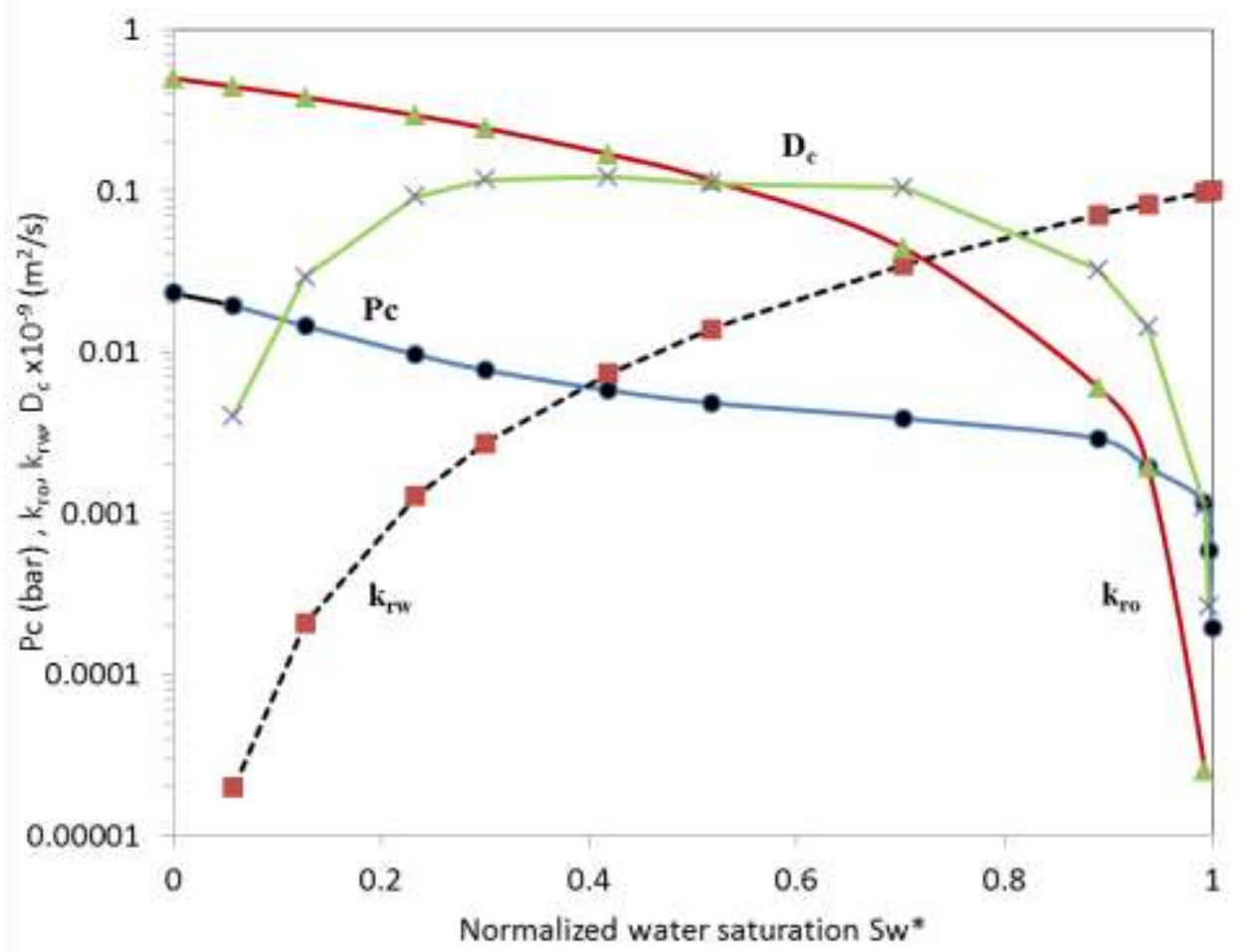

Figure 4: Calculation of the capillary diffusion coefficient $D_{c}$ using equation 2 taking typical $k_{r}$ curves and an empirical imbibition capillary pressure curve.

\section{RESULTS}

All the measured desaturation curves are shown in Figure 5. The initial and final water saturation varies respectively from $8 \%$ to $17 \%$ and $48 \%$ to $54 \%$, the variation of saturation being however similar for all samples (44\%, Table 2). Data points were also collected after $150 \mathrm{~h}$ (up to $350 \mathrm{~h}$, not shown) allowing to observe a very stable saturation. At the end of the tests, samples were removed from the glass tubes, oil droplets sticking on the surface carefully wiped off, and oil saturation measured again. Hence we could verify that the cut-off approach mentioned above gives a reasonable estimate of the true saturation inside the sample; indeed the oil saturation inside (with droplets and signal correction) and outside (without droplets) the tube could differ by at most 4 saturation units (4\%), while the contribution of the components above $100 \mathrm{~ms}$ can be much larger.

A careful examination of the curves reveals some differences when comparing data points $1 \mathrm{a}$ and $1 \mathrm{~b}$, and $2 \mathrm{a}$ and $2 \mathrm{~b}$ : oil flows out faster from the samples without alkaline salt (samples 1a and 2a) than from the ones with alkali. That difference becomes more obvious when fitting the data with the capillary diffusion model. 


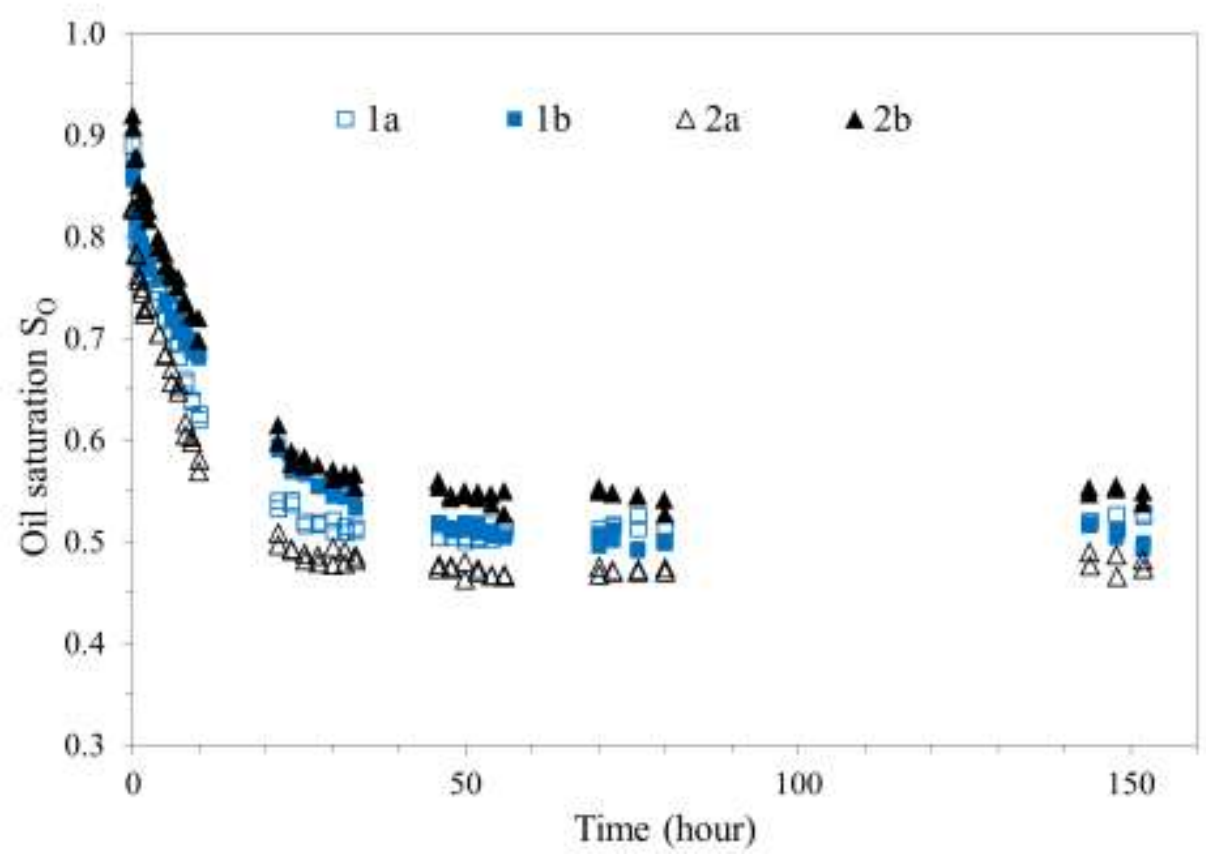

Figure 5: Desaturation curves obtained for all samples. Imbibition experiments on samples 1a and 2a was performed with the injection brine, and $1 \mathrm{~b}$ and $2 \mathrm{~b}$ with the alkaline brine.

We fitted the 4 data curves with the diffusion model (equation 3) by scaling the oil saturation between 1 and 0 (Figure 6), the asymptotic oil saturation at infinite time was chosen as the value measured outside the tube and is indicated as a horizontal line). First, we note that the experimental kinetic trend is consistent with a diffusion approach of the imbibition process. That result was not expected a priori because $\lambda_{w}, \lambda_{o}$ and $\lambda_{t}$ are functions of the saturation that is varying both with time and with position within the core, thus yielding potentially a non-unique coefficient $D_{c}$. Second the capillary diffusion coefficients $D_{c}$ can be split into two groups: the ones referring to imbibition without alkali and the ones referring to imbibition in alkaline brine. $D_{c}$ values without alkali are between 1.5 and 2 times larger than $D_{c}$ values with alkaline brine. That difference reflects qualitative observations that oil desaturation during the first 50 hours is slower with the alkaline brine than without alkaline. However, and despite these differences, the ultimate recovery reached after about 3 days of spontaneous imbibition, is similar whatever the brine type as already pointed out before. 

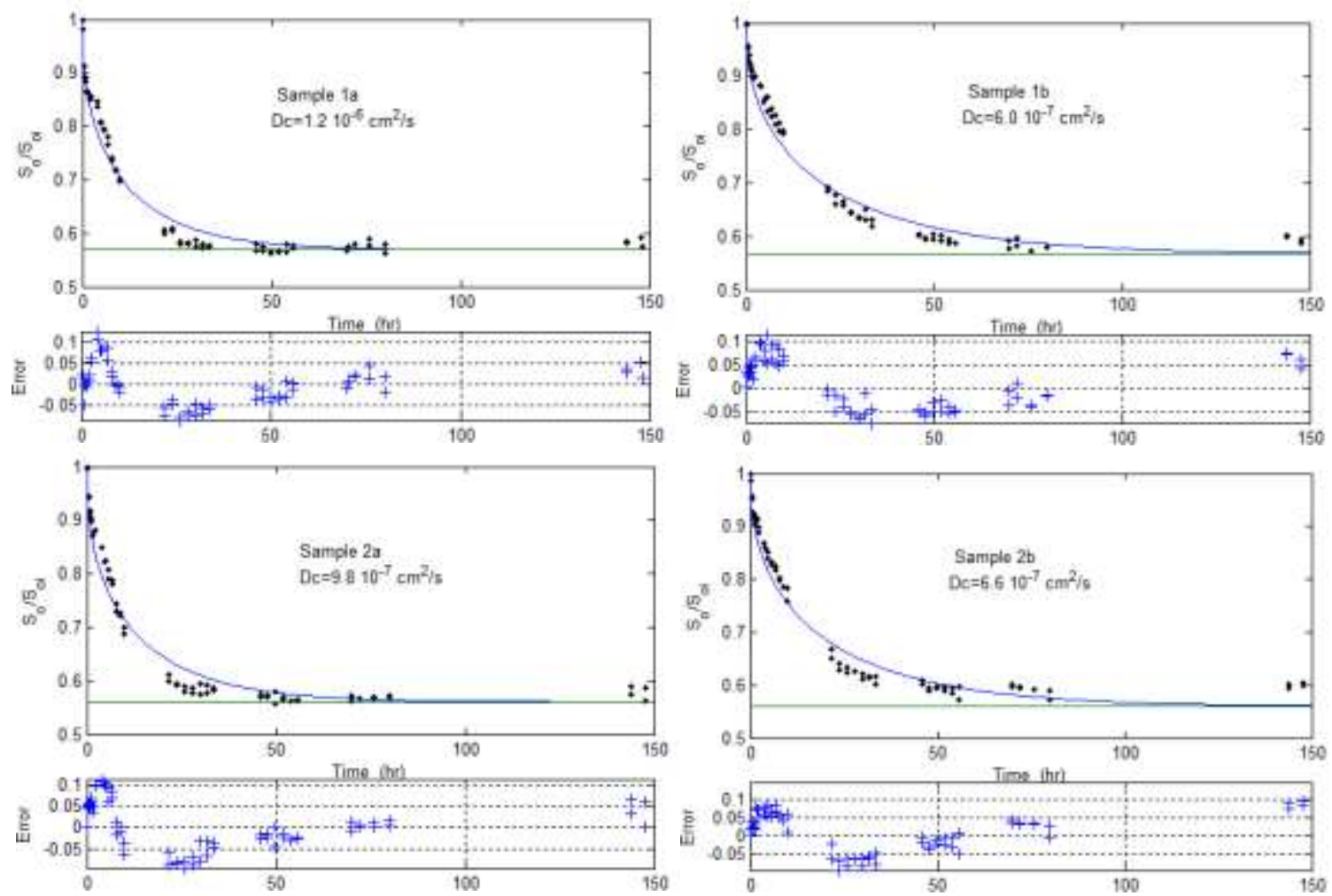

Figure 6: Desaturation curves fitted with the capillary diffusion model, equation 2.

\section{DISCUSSION}

Experimental data points are reasonably well fitted by a constant capillary diffusion model, indicating that the simplified equation can describe the kinetics of the process; the error is indeed mostly comprised within $\pm 5 \%$ (Figure 6 ). We discuss below three aspects that may have an impact on the kinetics of imbibition: sample size and flow geometry, modifications induced by the presence of alkali in solution, and the role played by other physical mechanisms than diffusion in the imbibition process.

\subsection{Scale and geometrical effects.}

The scaling of natural imbibition process has been studied since the sixties because of its determinant impact on the kinetics of oil recovery from water-wet fractured reservoirs subjected to a water drive. A compilation and thorough scale analysis of numerous spontaneous imbibition studies can be found in literature (Schmid and Geiger 2012). The significance of our experimental methodology based on NMR can be assessed by referring to fundamental scaling laws for infinite media (Marle 1981) and to the use of a general characteristic length that enables one to apply the scaling results on infinite media to finite media of various sizes and shapes (Shouxiang et al. 1997). Underlying basic principles are recalled hereafter. Dimension analysis of two-phase flow equations applied to 1D capillary imbibition shows that the imbibed water (or expelled oil) volume varies as the square root of time and that time for recovering a given fraction of the oil in place is proportional to the square of porous medium length. That scaling rule stands for infinite-acting imbibition situations, i.e. as long as the imbibing solution has not reached the limits of the sample, i.e. its center or its no-flow boundary if any. For finite-size 
samples of various shapes and for other flow geometries and boundary conditions, a characteristic length has to be defined in order to apply the above kinetics scaling rule.

A generalized characteristic length, $L_{c}$, can be defined (Shouxiang et al. 1997) following the concept of shape factor (Warren and Root 1963) applied to fractured reservoir simulation (Kazemi et al. 2013). Shape factor is defined on the basis of a simplified representation of the 3D exchange between a matrix block and surrounding fractures, wherein the exchange flux is proportional to the ratio of exchange surface area to the distance to block center or no-flow boundary, with a summation for all faces and a normalization to total block volume. Then, Shouxiang et al.'s definition of characteristic length is as follows:

$$
L_{c}=\sqrt{\frac{V}{\sum_{i=1}^{n} \frac{A_{i}}{X_{i}}}}
$$

with $V$ the bulk volume of the sample, $A_{i}$ the surface area of face $i$ open to flow and $X_{i}$ the distance between that face and the centre of the sample or the no-flow boundary if some faces are not open to flow. For our cylindrical core of length $L$ and diameter $D$, with all faces open, above definition yields:

$$
L_{c}=\frac{L D}{2 \sqrt{2 L^{2}+D^{2}}}
$$

Another definition of the characteristic length for the cylindrical sample is also proposed (Stoll et al. 2008), based on a summation of all faces contributions to recovery during the infinite-acting period, while taking into account the respective characteristic lengths that are L/2 for the 1D linear exchange through end faces, and D/4 for the exchange through lateral face according to the analytical solution at early times for that 2D radial flow geometry (Carslaw and Jaeger 1959):

$$
L_{c}=\frac{L D}{2 D+4 L}
$$

Above definitions of $L_{c}$ remain approximations of the characteristic length to be used for scaling the recovery kinetics of samples during the initial infinite-acting period of the spontaneous imbibition process. Furthermore, the end of the infinite-acting period occurs for various saturation states (i.e. various values of the average oil recovery) depending on the flow geometry (1D, 2D or 3D), the shape of sample and maybe also fluid properties (viscosities in particular). However, the kinetics of imbibition can be fairly well scaled from one sample size to another, provided that a given definition of characteristic length is adopted. Regarding our experiments, spontaneous imbibition duration is comprised between 50 and 100 hours for the mini-plugs of diameter D 15mm and length L 18mm (Figure 6). For standard plug size (D 40mm, L 60mm), that duration is multiplied by a factor of 8 , using either Shouxiang's or Stoll's definition.

Similarly, we used Eqs. 3 to 5 to calculate the required time to imbibe the same miniplug and standard plug, and found the same duration ratio of 1 to 8 . In addition to the small size of samples, the 3D flow geometry was also responsible for the short duration of our spontaneous imbibition tests, by comparison with 1D experiments on laterally-coated samples exchanging fluids only by one end or two opposite ends. Hence, the experimental set-up used in this study is quite efficient for a rapid screening of imbibition mechanisms influenced or not by chemicals. 


\subsection{Impact of alkali on spontaneous imbibition.}

One has still to explain the kinetic difference between alkaline brine and injection brine (Table 2, $\mathrm{D}_{\mathrm{c}}$ column). To that end, one has to consider the modification of surface and interfacial properties of the rock-fluid system in the presence of alkali. If one assumes a fixed wettability of the rockfluid system, then the decrease of water-oil IFT in the presence of alkali reduces the imbibition kinetics in the same ratio because the intensity of the driving capillary forces is proportional to the capillary pressure gradient (equation 2) and therefore to the interfacial tension. Herein, the IFT is reduced by a factor close to 10 (Figure 1), however the imbibition kinetics as quantified by the capillary diffusion coefficient $D_{c}$ is reduced by a factor not exceeding 2 . To explain that apparent discrepancy, one may assume that a rock wettability change was induced under alkaline conditions (Hirasaki and Zhang 2004), resulting in an increase of the driving capillary pressure by a factor equal to 5 . That wettability alteration would impact the intensity of capillary pressure but would not change the residual oil after imbibition as shown by Figure 6 and Table 2. That interpretation remains qualitative and still raises questions that would deserve a dedicated study to investigate the impact of alkali on the wettability properties of the rock under consideration.

\subsection{Insight into the mechanisms driving chemically-enhanced imbibition processes.}

The time scales of various processes involved in spontaneous imbibition are examined. First, the change of interfacial tension as the alkaline solution imbibes can be considered as instantaneous, as shown by measurements (Figure 1). Second, the local pore-scale changes of wettability should also be fast compared to the imbibition kinetics at porous medium sample scale. Third, the kinetics of diffusion of chemical additive into the porous medium is driven by the value of the effective molecular diffusion $\mathrm{D}_{\mathrm{e}}$ of the additive into the in-situ connate brine. $\mathrm{D}_{\mathrm{e}}$ can be estimated using Archie relationships as $D_{e} / D_{m} \sim \Phi S w(m=n=2)$. Hence, $D_{e}$ is initially 100 times smaller than the bulk molecular diffusion coefficient of water. Then, the estimated $D_{e}$ value is in the order of

$3 \times 10^{-11} \mathrm{~m}^{2} / \mathrm{s}$, which is significantly smaller than the measured coefficient, $D_{c}$, in the order of $10^{-10} \mathrm{~m}^{2} / \mathrm{s}$. In reality, the kinetics of imbibition is not primarily driven by a molecular diffusion mechanism, but by capillary suction mechanism, as proven by the capacity of the same samples to imbibe brine efficiently in the absence of alkali. One may then assume that the presence of alkali only enhances the intensity of capillary forces. This behavior stems from the initial wettability of studied samples, which remained preferentially water-wet after aging. Oil-wet miniplugs would not have imbibed any brine in the absence of any chemical additive, and the presence of additive altering rock wettability would probably have induced some imbibition but with a slower kinetics, as suggested by the chemical imbibition of oil-wet samples reported in another study (Bourbiaux et al. 2016). In the latter study, a very small pressure gradient applied between core ends was shown to greatly enhance the kinetics of chemical imbibition. In the present work, the capillary imbibition mechanism itself, instead of external viscous forces, drives the additive into the porous medium. In summary, in the context of fractured reservoirs, the enhancement of oil production by chemical additives does not solely rely on the molecular diffusion of chemicals that is very slow, but mainly depends on the synergy exerted by brine transfer mechanisms into matrix blocks, which may result from buoyancy, viscous drive due to fracture flow, and/or capillary imbibition as in our study. Indeed, the experimental methodology proposed herein is dedicated to fractured situations where the capillary imbibition is the predominant mechanism. 


\section{CONCLUSION}

A rapid and efficient methodology has been implemented to evaluate the EOR efficiency of chemicals from NMR measurements of spontaneous imbibition of chemical solutions on small cores. Results are shown for a reservoir rock-fluid system and an alkaline brine injection process. The main outcomes are threefold, concerning the experimental procedure, results analysis and application to the alkaline EOR process under consideration.

Thanks to a dedicated implementation of NMR technique, small samples $(\mathrm{D}=15 \mathrm{~mm}$ and $\mathrm{L}=$ $18 \mathrm{~mm}$ ) can be used without any loss of accuracy on the oil recovery measurements. That possibility to quantify spontaneous imbibition on so-small samples offers interesting perspectives regarding the preliminary assessment and selection of chemical EOR methods in fractured reservoirs.

Regarding results analysis of such experiments, a rapid screening of measured kinetics can be performed on the basis of a constant capillary "diffusion" coefficient characterizing the overall trend of the spontaneous imbibition process.

Application to the imbibition of restored reservoir samples under native (neutral) or alkaline conditions showed different kinetics that cannot be interpreted by the sole reduction of the brineoil interfacial tension in the presence of the alkaline solution. A modification of the rock wettability under alkaline conditions was then invoked to explain the different kinetics measured for that rock-fluid system. Further interpretation would require a dedicated study including a characterization of the wettability properties of the rock-fluids systems and a numerical study of their dynamic evolution during imbibition, beyond the scope of this article.

To conclude, the proposed methodology offers interesting opportunities to pre-select chemicals that enhance the contribution of imbibition to oil production, in particular in the case of lowpermeability fractured reservoirs.

\section{REFERENCES References}

Alyafei, N., Al-Menhali, A., Blunt, M.J.: Experimental and Analytical Investigation of Spontaneous Imbibition in Water-Wet Carbonates Transp Porous Med 115(1), 189-207 (2016)

Alyafei, N., Blunt, M.J.: Estimation of relative permeability and capillary pressure from mass imbibition experiments Advances in Water Resources 115, 88-94 (2018)

Berne, P., Bachaud, P., Fleury, M.: Diffusion Properties of Carbonated Caprocks from the Paris Basin Oil Gas Sci. Technol. - Rev. IFP 65(3), 473-484 (2010)

Bourbiaux, B., Fourno, A., Nguyen, Q.-L., Norrant, F., Robin, M., Rosenberg, E., Argillier, J.-F.: Experimental and Numerical Assessment of Chemical Enhanced Oil Recovery in Oil-Wet Naturally Fractured Reservoirs SPE Journal 21(03), 706-719 (2016)

Bourbiaux, B.J., Kalaydjian, F.J.: Experimental Study of Cocurrent and Countercurrent Flows in Natural Porous Media SPE Reservoir Engineering 5(03), 361-368 (1990)

Campbell, T.C., Krumrine, P.H.: Laboratory Studies On Alkaline Waterflooding. In: SPE Annual Technical Conference and Exhibition. Society of Petroleum Engineers (1979)

Carslaw, H.S., Jaeger, J.C.: Conduction of heat in solids. Oxford Science Publications (1959)

Chabert, M., Morvan, M., Tabary, R.: Fractured Carbonates. A Methodology to Evaluate Surfactant Performances. In: SPE Improved Oil Recovery Symposium. Society of Petroleum Engineers (2010) 
Chavent, G., Jaffre, J.: Mathematical models and finite elements for reservoir simulation. Single phase, multiphase and multicomponent flows through porous media. Studies in mathematics and its applications, vol. 17. North-Holland, Amsterdam [etc.] (1986)

Chevalier, T., Labaume, J., Gautier, S., Chevallier, E., Chabert, M.: A Novel Experimental Approach for Accurate Evaluation of Chemical EOR Processes in Tight Reservoir Rocks. In: SPE Improved Oil Recovery Conference. Society of Petroleum Engineers (2018)

Cooke, C.E., Williams, R.E., Kolodzie, P.A.: Oil Recovery by Alkaline Waterflooding Journal of Petroleum Technology 26(12), 1365-1374 (1974)

Crank, J.: The mathematics of diffusion, 2nd edn. Clarendon Press, Oxford (1976)

Ehrlich, R., Hasiba, H.H., Raimondi, P.: Alkaline Waterflooding for Wettability AlterationEvaluating a Potential Field Application Journal of Petroleum Technology 26(12), 1335-1343 (1974)

Foley, A.Y., Nooruddin, H.A., Blunt, M.J.: The impact of capillary backpressure on spontaneous counter-current imbibition in porous media Advances in Water Resources 107, 405-420 (2017)

Hamon, G., Vidal, J.: Scaling-Up the Capillary Imbibition Process From Laboratory Experiments on Homogeneous and Heterogeneous Samples. In: European Petroleum Conference. Society of Petroleum Engineers (1986)

Haugen, Å., Fernø, M.A., Mason, G., Morrow, N.R.: Capillary pressure and relative permeability estimated from a single spontaneous imbibition test Journal of Petroleum Science and Engineering 115, 66-77 (2014)

Hirasaki, G., Zhang, D.L.: Surface Chemistry of Oil Recovery From Fractured, Oil-Wet, Carbonate Formations SPE Journal 9(02), 151-162 (2004)

Kazemi, H., Merrill, L.S., Porterfield, K.L., Zeman, P.R.: Numerical Simulation of Water-Oil Flow in Naturally Fractured Reservoirs Society of Petroleum Engineers Journal 16(06), 317326 (2013)

March, R., Doster, F., Geiger, S.: Accurate early-time and late-time modeling of countercurrent spontaneous imbibition Water Resour. Res. 52(8), 6263-6276 (2016)

Marle, C.: Mulitphase flow in porous media. Technip (1981)

Mattax, C.C., Kyte, J.R.: Imbibition Oil Recovery from Fractured, Water-Drive Reservoir Society of Petroleum Engineers Journal 2(02), 177-184 (1962)

Morrow, N., Buckley, J.: Improved Oil Recovery by Low-Salinity Waterflooding Journal of Petroleum Technology 63(05), 106-112 (2013)

Ruth, D.W., Li, Y., Mason, G., Morrow, N.R.: An Approximate Analytical Solution for CounterCurrent Spontaneous Imbibition Transp Porous Med 66(3), 373-390 (2007)

Schmid, K.S., Alyafei, N., Geiger, S., Blunt, M.J.: Analytical Solutions for Spontaneous Imbibition. Fractional-Flow Theory and Experimental Analysis SPE Journal 21(06), 23082316 (2016)

Schmid, K.S., Geiger, S.: Universal scaling of spontaneous imbibition for water-wet systems Water Resour. Res. 48(3), 83 (2012)

Sheng, J.J.: Critical review of low-salinity waterflooding Journal of Petroleum Science and Engineering 120, 216-224 (2014)

Shouxiang, M., Morrow, N.R., Zhang, X.: Generalized scaling of spontaneous imbibition data for strongly water-wet systems Journal of Petroleum Science and Engineering 18(3-4), 165-178 (1997) 
Stoll, M., Hofman, J., Ligthelm, D.J., Faber, M.J., van den Hoek, P.: Toward Field-Scale Wettability Modification-The Limitations of Diffusive Transport SPE Reservoir Evaluation \& Engineering 11(03), 633-640 (2008)

Tecklenburg, J., Neuweiler, I., Dentz, M., Carrera, J., Geiger, S., Abramowski, C., Silva, O.: A non-local two-phase flow model for immiscible displacement in highly heterogeneous porous media and its parametrization Advances in Water Resources 62, 475-487 (2013)

Warren, J.E., Root, P.J.: The behavior of naturally fractured reservoirs SPE Journal, 244-255 (1963)

Zhou, Q., Oldenburg, C.M., Spangler, L.H., Birkholzer, J.T.: Approximate solutions for diffusive fracture-matrix transfer. Application to storage of dissolved CO 2 in fractured rocks Water Resour. Res. 53(2), 1746-1762 (2017) 